\title{
Violat: Generating Tests of Observational Refinement for Concurrent Objects
}

\author{
Michael Emmi ${ }^{1(\bowtie)}$ and Constantin Enea ${ }^{2}$ \\ 1 SRI International, New York, NY, USA \\ michael.emmi@sri.com \\ 2 Université de Paris, IRIF, CNRS, \\ 75013 Paris, France \\ cenea@irif.fr
}

\begin{abstract}
High-performance multithreaded software often relies on optimized implementations of common abstract data types (ADTs) like counters, key-value stores, and queues, i.e., concurrent objects. By using fine-grained and non-blocking mechanisms for efficient inter-thread synchronization, these implementations are vulnerable to violations of ADTconsistency which are difficult to detect: bugs can depend on specific combinations of method invocations and argument values, as well as rarely-occurring thread interleavings. Even given a bug-triggering interleaving, detection generally requires unintuitive test assertions to capture inconsistent combinations of invocation return values.

In this work we describe the Violat tool for generating tests that witness violations to atomicity, or weaker consistency properties. Violat generates self-contained and efficient programs that test observational refinement, i.e., substitutability of a given ADT with a given implementation. Our approach is both sound and complete in the limit: for every consistency violation there is a failed execution of some test program, and every failed test signals an actual consistency violation. In practice we compromise soundness for efficiency via random exploration of test programs, yielding probabilistic soundness instead. Violat's tests reliably expose ADT-consistency violations using off-the-shelf approaches to concurrent test validation, including stress testing and explicit-state model checking.
\end{abstract}

\section{Introduction}

Many mainstream software platforms including Java and .NET support multithreading to enable parallelism and reactivity. Programming multithreaded code effectively is notoriously hard, and prone to data races on shared memory accesses, or deadlocks on the synchronization used to protect accesses. Rather than confronting these difficulties, programmers generally prefer to leverage libraries providing concurrent objects [19,29], i.e., optimized thread-safe implementations of common abstract data types (ADTs) like counters, key-value stores, and queues. For instance, Java's concurrent collections include implementations which eschew the synchronization bottlenecks associated with lock-based

(C) The Author(s) 2019

I. Dillig and S. Tasiran (Eds.): CAV 2019, LNCS 11562, pp. 534-546, 2019.

https://doi.org/10.1007/978-3-030-25543-5_30 
mutual exclusion, opting instead for non-blocking mechanisms [28] provided by hardware operations like atomic compare and exchange.

Concurrent object implementations are themselves vulnerable to elusive bugs: even with effective techniques for exploring the space of thread interleavings, like stress testing or model checking $[7,30,47]$, bugs often depend on specific combinations of method invocations and argument values. Furthermore, even recognizing whether a given execution is correct is non-trivial, since recognition generally requires unintuitive test assertions to identify inconsistent combinations of return values. Technically, correctness amounts to observational refinement $[18,21,32]$, which captures the substitutability of an ADT with an implementation [23]: any combination of values admitted by a given implementation is also admitted by the given ADT specification.

In this work we describe an approach to generating tests of observational refinement for concurrent objects, as implemented by the Violat tool, which we use to discover violations to atomicity (and weaker consistency properties) in widely-used concurrent objects $[9,10,12]$. Unlike previous approaches based on linearizability $[4,20,46]$, Violat generates self-contained test programs which do not require enumerating linearizations dynamically per execution, instead statically precomputing the ADT-admitted return-value outcomes per test program, once, prior to testing. Despite this optimization, the approach is both sound and complete, i.e., in the limit: for every consistency violation there is a failed execution of some test program, and every failed test witnesses an actual consistency violation. In practice, we compromise soundness for efficiency via random exploration of test programs, achieving probabilistic soundness instead.

Besides improving the efficiency of test execution, Violat's self-contained tests can be validated by both stress testers and model checkers, and double as regression and conformance tests. Our previous works $[9,10,12]$ demonstrate that Violat's tests reliably expose ADT-consistency violations in Java implementations using the Java Concurrency Stress testing tool [42]. In particular, Violat has uncovered atomicity violations in over 50 methods from Java's concurrent collections; many of these violations seem to correspond with their documentations' mention of weakly-consistent behavior, while others indicate confirmed implementation bugs, which we have reported.

Previous work used Violat in empirical studies, without artifact evaluation $[9,10,12]$. This article is the first to consider Violat itself for evaluation, the first to describe its implementation and usage, and includes several novel extensions. For instance, in addition to stress testing, Violat now includes an integration with Java Pathfinder [47]; besides enabling complete systematic coverage of a given test program, this integration enables the output of the execution traces leading to consistency violations, thus facilitating diagnosis and repair. Furthermore, Violat is now capable of generating tests of any user-provided implementation, in addition to those distributed with Java. 


\section{Overview of Test Generation with Violat}

Violat generates self-contained programs to test the observational refinement of a given concurrent object implementation with respect to its abstract data type (ADT), according to Fig. 1. While its methodology is fairly platform agnostic, Violat currently integrates with the Java platform. Accordingly, its input includes the fully-qualified name of a single Java class, which is assumed to be available either on the system classpath, or in a user-provided Java archive (JAR); its output is a sequence of Java classes which can be tested with offthe-shelf back-end analysis engines, including the Java Concurrency Stress testing tool [42] and Java Pathfinder [47]. Our current implementation integrates directly with both back-ends, and thus reports test results directly, signaling any discovered consistency violations.

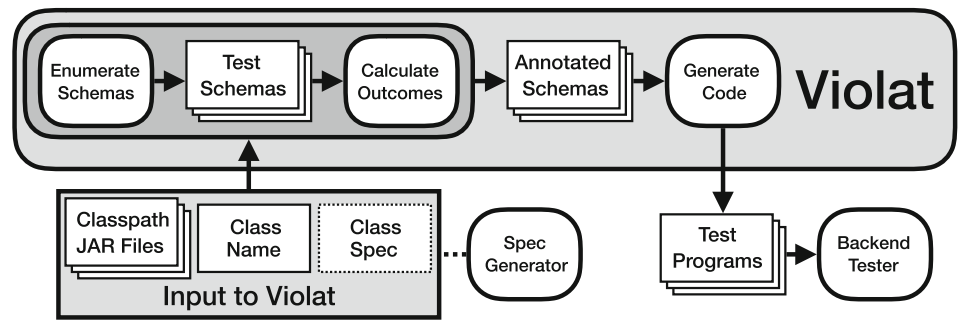

Fig. 1. Violat generates tests by enumerating program schemas invoking a given concurrent object, annotating those schemas with the expected outcomes of invocations according to ADT specifications, and translating annotated schemas to executable tests.

Violat generates tests according to a three-step pipeline. The first step, described in Sect. 3, enumerates test program schemas, i.e., concise descriptions of programs as parallel sequences of invocations of the given concurrent object's methods. For example, Fig. 2 lists several test schemas for Java's ConcurrentHashMap. The second step, described in Sect.4, annotates each schema with a set of expected outcomes, i.e., the combinations of return values among the given schema's invocations which are admitted according to the given object's ADT specification. The final step, described in Sect. 5, translates each schema into a self-contained ${ }^{1}$ Java class.

Technically, to guide the enumeration of schemas and calculation of outcomes, Violat requires a specification of the given concurrent object, describing constructor and method signatures. While this could be generated automatically from the object's bytecode, our current implementation asks the user to input this specification in JSON format. By additionally indicating whether methods are read-only or weakly-consistent, the user can provide additional hints to

\footnotetext{
1 The generated class imports only a given concurrent object, and a few basic java.util classes.
} 
improve schema enumeration and outcome calculation. For instance, excessive generation of programs with only read-only methods is unlikely to uncover consistency violations, and weakly-consistent ADT methods generally allow additional outcomes - see Emmi and Enea [12]. Furthermore, Violat attempts to focus the blame for discovered violations by constructing tests with a small number of specified untrusted methods, e.g., just one.

\section{Test Enumeration}

To enumerate test programs effectively, Violat considers a simple representation of program schemas, as depicted in Fig. 2. We write schemas with a familiar notation, as parallel compositions $\{\ldots\}||\{\ldots\}$ of method-invocation sequences. Intuitively, schemas capture parallel threads invoking sequences of methods of a given concurrent object. Besides the parallelism, these schemas include only trivial control and data flow. For instance, we exclude conditional statements and loops, as well as passing return values as arguments, in favor of straight-line code with literal argument values. Nevertheless, this simple notion is expressive enough to capture any possible outcome, i.e., combination of invocation return values, of programs with arbitrarily complex control flow, data flow, and synchronization. To see this, consider any outcome $\boldsymbol{y}$ admitted by some execution of a program with arbitrarily-complex control and data flow in which methods are invoked with argument values $\boldsymbol{x}$, collectively. The schema in which each thread invokes the same methods of a thread of the original program with literal values $\boldsymbol{x}$, collectively, is guaranteed to admit the same outcome $\boldsymbol{y}$.

\begin{tabular}{|c|c|}
\hline \multicolumn{2}{|l|}{ java.util.ConcurrentHashMap } \\
\hline Schema / Method & Outcome \\
\hline$\{\operatorname{put}(0,0) ; \operatorname{put}(1,1) ; \operatorname{put}(1,1)\} \|\{\operatorname{put}(0,1) ; \operatorname{clear}()\}$ & $N, N, N, N,()$ \\
\hline$\{\operatorname{put}(0,0) ; \operatorname{remove}(1)\} \|\{\operatorname{put}(1,0) ; \operatorname{contains}(\theta)\}$ & $N, 0, N, F$ \\
\hline$\{\operatorname{get}(1) ; \operatorname{containsValue}(1)\} \|\{\operatorname{put}(1,1) ; \operatorname{put}(0,1) ; \operatorname{put}(1,0)\}$ & \} $1, F, N, N, 1$ \\
\hline$\{\operatorname{put}(\theta, 1) ; \operatorname{put}(1,0)\} \|\{$ elements ()$\}$ & $N, N,[0]$ \\
\hline$\{\operatorname{put}(0,1) ; \operatorname{put}(1,0)\} \|\{\operatorname{entrySet}()\}$ & $N, N,[1=0]$ \\
\hline$\{\operatorname{put}(1,1)\} \|\{\operatorname{put}(1,2) ; \operatorname{isEmpty}()\}$ & $\mathrm{N}, 1, \mathbf{T}$ \\
\hline$; \operatorname{put}(1,1)\} \|\{\operatorname{keySet}()\}$ & [1] \\
\hline$\{\operatorname{keys}()\} \|\{\operatorname{put}(0,1) ; \operatorname{put}(1,1)\}$ & {$[1], N, N$} \\
\hline$\{\operatorname{put}(1,0) ; \operatorname{put}(1,1) ; \operatorname{mappingCount}()\} \|\{\operatorname{remove}(1)\}$ & $\mathrm{N}, \mathrm{N}, 2,0$ \\
\hline$\{\operatorname{put}(1,0) ; \operatorname{put}(1,1) ; \operatorname{size}()\} \|\{\operatorname{remove}(1)\}$ & $N, N, 2,0$ \\
\hline$\{\operatorname{put}(0,1) ; \operatorname{put}(1,1)\} \|\{\operatorname{toString}()\}$ & $N, N, 1=1$ \\
\hline$\{\operatorname{put}(0,1) ; \operatorname{put}(1,0)\} \|\{\operatorname{values}()\}$ & $N, N,[0]$ \\
\hline
\end{tabular}

Fig. 2. Program schemas generated by Violat for Java's ConcurrentHashMap class, along with outcomes which are observed in testing, yet not predicated by Violat.

For a given concurrent object, Violat enumerates schemas according to a few configurable parameters, including bounds on the number of threads, 
invocations, and (primitive) values. By default, Violat generates schemas with exactly 2 threads, between 3 and 6 invocations, and exactly 2 values. While our initial implementation enumerated schemas systematically according to a welldefined order, empirically we found that this strategy spends too much time in neighborhoods of uninteresting schemas, i.e., which do not expose violations. Ultimately we adopted a pseudorandom enumeration which constructs each schema independently by randomly choosing the number of threads, invocations, and values, within the given parameter bounds, and randomly populating threads with invocations. Methods are selected according to a weighted random choice, in which the weights of read-only and untrusted methods is 1 ; trusted mutator methods have weight 3 . The read-only and trusted designations are provided by class specifications - see Sect. 2. Integer argument values are chosen randomly between 0 and 1 , according to the default value bound; generic-typed arguments are assumed to be integers. Collection and map values are constructed from randomly-chosen integer values, up to size 2. In principle, all of these bounds are configurable, but we have found these defaults to work reasonably well.

Note that while the manifestation of a given concurrency bug can, in principle, rely on large bounds on threads, invocations, and values, recent studies demonstrate that the majority $(96 \%)$ can be reproduced with just 2 threads [25]. Furthermore, while our current implementation adheres to the simple notion of schema in which all threads are execute in parallel, Violat can easily be extended to handle a more complex notion of schema in which threads are partially ordered, thus capturing arbitrary program synchronization. Nevertheless, this simple notion seems effective at exposing violations without requiring additional synchronization - see Emmi and Enea [12, Section 5.2].

\section{Computing Expected Outcomes}

To capture violations to observational refinement, Violat computes the set of expected outcomes, i.e., those admitted by a given concurrent object's abstract data type (ADT), for each program schema. Violat essentially follows the approach of Line-Up [4] by computing expected outcomes from sequential executions of the given implementation. While this approach assumes that the sequential behavior of a given implementation does adhere to its implicit ADT specification - and that the outcomes of concurrent executions are also outcomes of sequential executions - there is typically no practical alternative, since behavioral ADT specifications are rarely provided.

Violat computes the expected outcomes of a given schema once, by enumerating all possible shuffles of threads' invocations, and recording the return values of each shuffle when executed by the given implementation. For instance, there are 10 ways to shuffle the threads of the schema

$$
\{\operatorname{get}(1) ; \text { containsValue(1) }\} \|\{\operatorname{put}(1,1) ; \operatorname{put}(0,1) ; \operatorname{put}(1,0)\}
$$

from Fig. 2, including the sequence

get (1); put (1,1); put $(0,1) ; \operatorname{put}(1,0) ;$ containsValue(1). 


\begin{tabular}{|c|c|c|c|}
\hline \multicolumn{4}{|c|}{ ConcurrentHashMap: containsValue } \\
\hline outcome & atomic? & paths (JPF) & frequency (jcstress) \\
\hline 0 , true, null, null, 1 & $\checkmark$ & 3 & 13,287 \\
\hline 1, false, null, null, 1 & $x$ & 3 & 2 \\
\hline 1, true, null, null, 1 & $\checkmark$ & 3 & 16,417 \\
\hline null, false, null, null, 1 & $\checkmark$ & 6 & $3,638,600$ \\
\hline null, true, null, null, 1 & $\checkmark$ & 3 & 9,504 \\
\hline
\end{tabular}

Fig. 4. Observed outcomes for the size method, recorded by Java Pathfinder and jcstress. Outcomes list return values in program-text order, e.g., get's return value is listed first.

\section{Code Generation and Back-End Integrations}

Once schemas are annotated with expected outcomes, the translation to actual test programs is fairly straightforward. Note that until this point, Violat is mainly agnostic to the underlying platform for which tests are being generated. The only exception is in computing the expected outcomes for schema linearizations, which executes the given concurrent object implementation as a stand-in oracle for its implicit ADT specification.

Figure 3 lists a simplification of the code generated for the containsValue schema of Fig. 2. The test program initializes a concurrent-object instance and a hash table of expected outcomes, then runs the schema's threads in parallel, recording the results of each invocation, and checks, after threads complete, whether the recorded outcome is expected. To avoid added inter-thread interference and the masking of potential weak-memory effects, each recorded result is isolated to a distinct cache line via Java's contended decorator. The actual generated code also includes exception handling, elided here for brevity.

Our current implementation of Violat integrates with two analysis back-ends: the Java Concurrency Stress testing tool [42] (jcstress) and Java Pathfinder [47]. Figure 4 demonstrates the results of each tool on the code generated from the containsValue schema of Fig. 2. Each tool observes executions with the 4 expected outcomes, as well as executions yielding an outcome that Violat does not predict, thus signaling a violation to observational refinement (and atomicity). Java Pathfinder explores 18 program paths in a few seconds - achieving exhaustiveness via partial-order reduction [16] - while jcstress explores nearly 4 million executions in $1 \mathrm{~s}$, observing the unpredicted outcome only twice. Aside from this example, Violat has uncovered consistency violations in over 50 methods of Java's concurrent collections $[9,10,12]$. 


\section{Usage}

Violat is implemented as a Node.js command-line application, available from GitHub and npm. ${ }^{2}$ Its basic functionality is provided by the command:

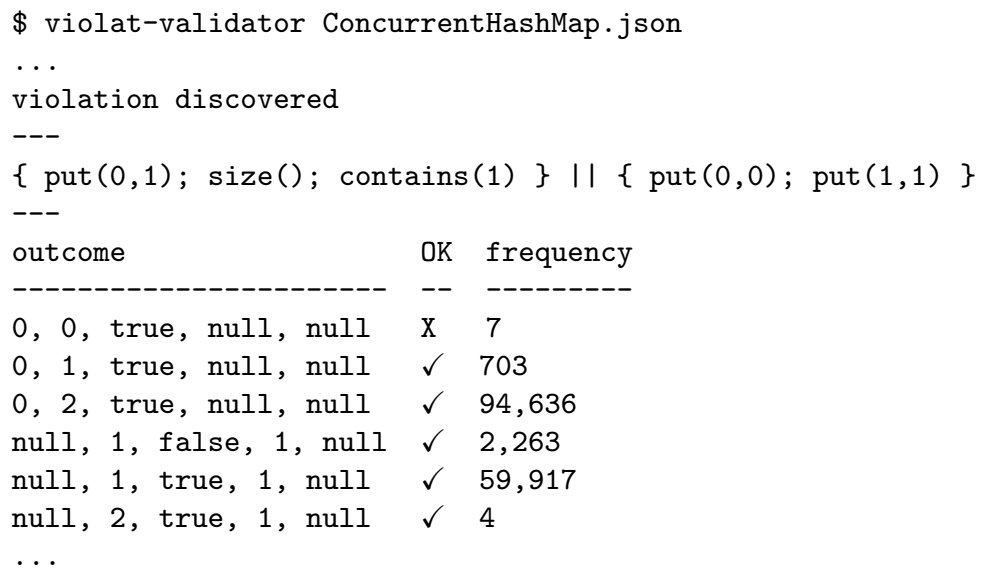

reporting violations among 100 generated programs. User-provided classes, individual schemas, program limits, and particular back-ends can also be specified:

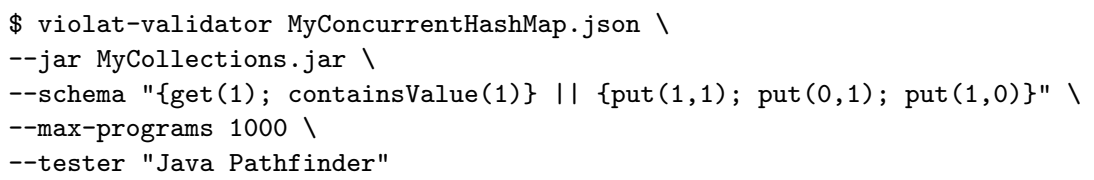

A full selection of parameters is available from the usage instructions:

$$
\text { \$ violat-validator --help }
$$

\section{Related Work}

Terragni and Pezzà survey several works on test generation for concurrent objects [45]. Like Violat, Ballerina [31] and ConTeGe [33] enumerate tests randomly, while ConSuite [43], AutoConTest [44], and CovCon [6] exploit static analysis to compute potential shared-memory access conflicts to reduce redundancy among generated tests. Similarly, Omen [35-38], Narada [40], Intruder [39], and Minion [41] reduce redundancy by anticipating potential concurrency faults during sequential execution. Ballerina [31] and ConTeGe [33] compute linearizations, but only identify generic faults like data races, deadlocks, and exceptions, being neither sound nor complete for testing observational refinement: fault-free executions with un-admitted return-value combinations are false negatives, while faulting executions with admitted return-value combinations are generally false positives - many non-blocking concurrent objects exhibit

\footnotetext{
${ }^{2}$ https://github.com/michael-emmi/violat.
} 
data races by design. We consider the key innovations of these works, i.e., redundancy elimination, orthogonal and complementary to ours. While Pradel and Gross do consider subclass substitutability [34], they only consider programs with two concurrent invocations, and require exhaustive enumeration of the superclass's thread interleavings to calculate admitted outcomes. In contrast, Violat computes expected outcomes without interleaving method implementations, i.e., considering them atomic.

Others generate tests for memory consistency. TSOtool [17] generates random tests against the total-store order (TSO) model, while LCHECK [5] employs genetic algorithms. Mador-Haim et al. [26,27] generate litmus tests to distinguish several memory models, including TSO, partial-store order (PSO), relaxedmemory order (RMO), and sequential consistency (SC). CppMem [2] considers the $\mathrm{C}++$ memory model, while Herd [1] considers release-acquire (RA) and Power in addition to the aforementioned models. McVerSi [8] employs genetic algorithms to enhance test coverage, while Wickerson et al. [48] leverage the Alloy model finder [22]. In some sense, these works generate tests of observational refinement for platforms implementing memory-system ADTs, i.e., with read and write operations, whereas Violat targets arbitrary ADTs, including collections with arbitrarily-rich sets of operations.

Violat more closely follows work on linearizability checking. Herlihy and Wing [20] established the soundness of linearizability for observational refinement, and Filipovic et al. [14] established completeness. Wing and Gong [49] developed a linearizability-checking algorithm, which was later adopted by LineUp [4] and optimized by Lowe [24]; while Violat pays the exponential cost of enumerating linearizations once per program, these approaches pay that cost per execution - an exponential quantity itself. Gibbons and Korach [15] established NP-hardness of per-execution linearizability checking for arbitrary objects, while Emmi and Enea [11] demonstrate tractability for collections. Bouajjani et al. [3] propose polynomial-time approximations, and Emmi et al. [13] demonstrate efficient symbolic algorithms. Finally, Emmi and Enea $[9,10,12]$ apply Violat to checking atomicity and weak-consistency of Java concurrent objects.

Acknowledgement. This work is supported in part by the European Research Council (ERC) under the European Union's Horizon 2020 research and innovation programme (grant No. 678177).

\section{References}

1. Alglave, J., Maranget, L., Tautschnig, M.: Herding cats: modelling, simulation, testing, and data mining for weak memory. ACM Trans. Program. Lang. Syst. 36(2), 7:1-7:74 (2014). https://doi.org/10.1145/2627752

2. Batty, M., Owens, S., Sarkar, S., Sewell, P., Weber, T.: Mathematizing C++ concurrency. In: Ball, T., Sagiv, M. (eds.) Proceedings of the 38th ACM SIGPLANSIGACT Symposium on Principles of Programming Languages, POPL 2011, Austin, TX, USA, 26-28 January 2011, pp. 55-66. ACM (2011). https://doi.org/ $10.1145 / 1926385.1926394$ 
3. Bouajjani, A., Emmi, M., Enea, C., Hamza, J.: Tractable refinement checking for concurrent objects. In: Rajamani, S.K., Walker, D. (eds.) Proceedings of the 42nd Annual ACM SIGPLAN-SIGACT Symposium on Principles of Programming Languages, POPL 2015, Mumbai, India, 15-17 January 2015, pp. 651-662. ACM (2015). https://doi.org/10.1145/2676726.2677002

4. Burckhardt, S., Dern, C., Musuvathi, M., Tan, R.: Line-up: a complete and automatic linearizability checker. In: Zorn, B.G., Aiken, A. (eds.) Proceedings of the 2010 ACM SIGPLAN Conference on Programming Language Design and Implementation, PLDI 2010, Toronto, Ontario, Canada, 5-10 June 2010, pp. 330-340. ACM (2010). https://doi.org/10.1145/1806596.1806634

5. Chen, Y., et al.: Fast complete memory consistency verification. In: 15th International Conference on High-Performance Computer Architecture (HPCA-15 2009), 14-18 February 2009, Raleigh, North Carolina, USA, pp. 381-392. IEEE Computer Society (2009). https://doi.org/10.1109/HPCA.2009.4798276

6. Choudhary, A., Lu, S., Pradel, M.: Efficient detection of thread safety violations via coverage-guided generation of concurrent tests. In: Uchitel, S., Orso, A., Robillard, M.P. (eds.) Proceedings of the 39th International Conference on Software Engineering, ICSE 2017, Buenos Aires, Argentina, 20-28 May 2017, pp. 266-277. IEEE/ACM (2017). https://doi.org/10.1109/ICSE.2017.32

7. Clarke, E.M., Grumberg, O., Peled, D.A.: Model Checking. MIT Press (2001). http://books.google.de/books?id=Nmc4wEaLXFEC

8. Elver, M., Nagarajan, V.: McVerSi: a test generation framework for fast memory consistency verification in simulation. In: 2016 IEEE International Symposium on High Performance Computer Architecture, HPCA 2016, Barcelona, Spain, 1216 March 2016, pp. 618-630. IEEE Computer Society (2016). https://doi.org/10. 1109/HPCA.2016.7446099

9. Emmi, M., Enea, C.: Exposing non-atomic methods of concurrent objects. CoRR abs/1706.09305 (2017). http://arxiv.org/abs/1706.09305

10. Emmi, M., Enea, C.: Monitoring weak consistency. In: Chockler, H., Weissenbacher, G. (eds.) CAV 2018. LNCS, vol. 10981, pp. 487-506. Springer, Cham (2018). https://doi.org/10.1007/978-3-319-96145-3_26

11. Emmi, M., Enea, C.: Sound, complete, and tractable linearizability monitoring for concurrent collections. PACMPL 2(POPL), 25:1-25:27 (2018). https://doi.org/10. $1145 / 3158113$

12. Emmi, M., Enea, C.: Weak-consistency specification via visibility relaxation. PACMPL 3(POPL), 60:1-60:28 (2019). https://dl.acm.org/citation.cfm?id= 3290373

13. Emmi, M., Enea, C., Hamza, J.: Monitoring refinement via symbolic reasoning. In: Grove, D., Blackburn, S. (eds.) Proceedings of the 36th ACM SIGPLAN Conference on Programming Language Design and Implementation, Portland, OR, USA, 15-17 June 2015, pp. 260-269. ACM (2015). https://doi.org/10.1145/2737924. 2737983

14. Filipovic, I., O’Hearn, P.W., Rinetzky, N., Yang, H.: Abstraction for concurrent objects. Theor. Comput. Sci. 411(51-52), 4379-4398 (2010). https://doi.org/10. 1016/j.tcs.2010.09.021

15. Gibbons, P.B., Korach, E.: Testing shared memories. SIAM J. Comput. 26(4), 1208-1244 (1997). https://doi.org/10.1137/S0097539794279614

16. Godefroid, P. (ed.): Partial-Order Methods for the Verification of Concurrent Systems. LNCS, vol. 1032. Springer, Heidelberg (1996). https://doi.org/10.1007/3540-60761-7 
17. Hangal, S., Vahia, D., Manovit, C., Lu, J.J., Narayanan, S.: TSOtool: a program for verifying memory systems using the memory consistency model. In: 31st International Symposium on Computer Architecture (ISCA 2004), 19-23 June 2004, Munich, Germany, pp. 114-123. IEEE Computer Society (2004). https://doi.org/ 10.1109/ISCA.2004.1310768

18. He, J., Hoare, C.A.R., Sanders, J.W.: Data refinement refined resume. In: Robinet, B., Wilhelm, R. (eds.) ESOP 1986. LNCS, vol. 213, pp. 187-196. Springer, Heidelberg (1986). https://doi.org/10.1007/3-540-16442-1_14

19. Herlihy, M., Shavit, N.: The Art of Multiprocessor Programming. Morgan Kaufmann, San Mateo (2008)

20. Herlihy, M., Wing, J.M.: Linearizability: a correctness condition for concurrent objects. ACM Trans. Program. Lang. Syst. 12(3), 463-492 (1990). https://doi. org $/ 10.1145 / 78969.78972$

21. Hoare, C.A.R., He, J., Sanders, J.W.: Prespecification in data refinement. Inf. Process. Lett. 25(2), 71-76 (1987). https://doi.org/10.1016/0020-0190(87)90224-9

22. Jackson, D.: Alloy: a lightweight object modelling notation. ACM Trans. Softw. Eng. Methodol. 11(2), 256-290 (2002). https://doi.org/10.1145/505145.505149

23. Liskov, B., Wing, J.M.: A behavioral notion of subtyping. ACM Trans. Program. Lang. Syst. 16(6), 1811-1841 (1994). https://doi.org/10.1145/197320.197383

24. Lowe, G.: Testing for linearizability. Concurrency Comput. Pract. Exp. 29(4) (2017). https://doi.org/10.1002/cpe.3928

25. Lu, S., Park, S., Seo, E., Zhou, Y.: Learning from mistakes: a comprehensive study on real world concurrency bug characteristics. In: Eggers, S.J., Larus, J.R. (eds.) Proceedings of the 13th International Conference on Architectural Support for Programming Languages and Operating Systems, ASPLOS 2008, Seattle, WA, USA, 1-5 March 2008, pp. 329-339. ACM (2008). https://doi.org/10.1145/ 1346281.1346323

26. Mador-Haim, S., Alur, R., Martin, M.M.K.: Generating litmus tests for contrasting memory consistency models. In: Touili, T., Cook, B., Jackson, P. (eds.) CAV 2010. LNCS, vol. 6174, pp. 273-287. Springer, Heidelberg (2010). https://doi.org/10. 1007/978-3-642-14295-6_26

27. Mador-Haim, S., Alur, R., Martin, M.M.K.: Litmus tests for comparing memory consistency models: how long do they need to be? In: Stok, L., Dutt, N.D., Hassoun, S. (eds.) Proceedings of the 48th Design Automation Conference, DAC 2011, San Diego, California, USA, 5-10 June 2011, pp. 504-509. ACM (2011). https://doi. org/10.1145/2024724.2024842

28. Michael, M.M., Scott, M.L.: Simple, fast, and practical non-blocking and blocking concurrent queue algorithms. In: Burns, J.E., Moses, Y. (eds.) Proceedings of the Fifteenth Annual ACM Symposium on Principles of Distributed Computing, Philadelphia, Pennsylvania, USA, 23-26 May 1996, pp. 267-275. ACM (1996). https://doi.org/10.1145/248052.248106

29. Moir, M., Shavit, N.: Concurrent data structures. In: Mehta, D.P., Sahni, S. (eds.) Handbook of Data Structures and Applications. Chapman and Hall/CRC (2004). https://doi.org/10.1201/9781420035179.ch47

30. Musuvathi, M., Qadeer, S.: CHESS: systematic stress testing of concurrent software. In: Puebla, G. (ed.) LOPSTR 2006. LNCS, vol. 4407, pp. 15-16. Springer, Heidelberg (2007). https://doi.org/10.1007/978-3-540-71410-1_2 
31. Nistor, A., Luo, Q., Pradel, M., Gross, T.R., Marinov, D.: Ballerina: automatic generation and clustering of efficient random unit tests for multithreaded code. In: Glinz, M., Murphy, G.C., Pezzè, M. (eds.) 34th International Conference on Software Engineering, ICSE 2012, 2-9 June 2012, Zurich, Switzerland, pp. 727-737. IEEE Computer Society (2012). https://doi.org/10.1109/ICSE.2012.6227145

32. Plotkin, G.D.: LCF considered as a programming language. Theor. Comput. Sci. 5(3), 223-255 (1977). https://doi.org/10.1016/0304-3975(77)90044-5

33. Pradel, M., Gross, T.R.: Fully automatic and precise detection of thread safety violations. In: Vitek, J., Lin, H., Tip, F. (eds.) ACM SIGPLAN Conference on Programming Language Design and Implementation, PLDI 2012, Beijing, China, 1116 June 2012, pp. 521-530. ACM (2012). https://doi.org/10.1145/2254064.2254126

34. Pradel, M., Gross, T.R.: Automatic testing of sequential and concurrent substitutability. In: Notkin, D., Cheng, B.H.C., Pohl, K. (eds.) 35th International Conference on Software Engineering, ICSE 2013, San Francisco, CA, USA, 18-26 May 2013, pp. 282-291. IEEE Computer Society (2013). https://doi.org/10.1109/ICSE. 2013.6606574

35. Samak, M., Ramanathan, M.K.: Multithreaded test synthesis for deadlock detection. In: Black, A.P., Millstein, T.D. (eds.) Proceedings of the 2014 ACM International Conference on Object Oriented Programming Systems Languages \& Applications, OOPSLA 2014, Part of SPLASH 2014, Portland, OR, USA, 20-24 October 2014, pp. 473-489. ACM (2014). https://doi.org/10.1145/2660193.2660238

36. Samak, M., Ramanathan, M.K.: Omen+: a precise dynamic deadlock detector for multithreaded java libraries. In: Cheung, S., Orso, A., Storey, M.D. (eds.) Proceedings of the 22nd ACM SIGSOFT International Symposium on Foundations of Software Engineering, (FS-22), Hong Kong, China, 16-22 November 2014, pp. 735-738. ACM (2014). https://doi.org/10.1145/2635868.2661670

37. Samak, M., Ramanathan, M.K.: Omen: a tool for synthesizing tests for deadlock detection. In: Black, A.P. (ed.) Conference on Systems, Programming, and Applications: Software for Humanity, SPLASH 2014, Portland, OR, USA, 20-24 October 2014, Companion Volume, pp. 37-38. ACM (2014). https://doi.org/10. $1145 / 2660252.2664663$

38. Samak, M., Ramanathan, M.K.: Trace driven dynamic deadlock detection and reproduction. In: Moreira, J.E., Larus, J.R. (eds.) ACM SIGPLAN Symposium on Principles and Practice of Parallel Programming, PPoPP 2014, Orlando, FL, USA, 15-19 February 2014, pp. 29-42. ACM (2014). https://doi.org/10.1145/2555243. 2555262

39. Samak, M., Ramanathan, M.K.: Synthesizing tests for detecting atomicity violations. In: Nitto, E.D., Harman, M., Heymans, P. (eds.) Proceedings of the 2015 10th Joint Meeting on Foundations of Software Engineering, ESEC/FSE 2015, Bergamo, Italy, 30 August-4 September 2015, pp. 131-142. ACM (2015). https:// doi.org/10.1145/2786805.2786874

40. Samak, M., Ramanathan, M.K., Jagannathan, S.: Synthesizing racy tests. In: Grove, D., Blackburn, S. (eds.) Proceedings of the 36th ACM SIGPLAN Conference on Programming Language Design and Implementation, Portland, OR, USA, 15-17 June 2015, pp. 175-185. ACM (2015). https://doi.org/10.1145/2737924. 2737998

41. Samak, M., Tripp, O., Ramanathan, M.K.: Directed synthesis of failing concurrent executions. In: Visser, E., Smaragdakis, Y. (eds.) Proceedings of the 2016 ACM SIGPLAN International Conference on Object-Oriented Programming, Systems, Languages, and Applications, OOPSLA 2016, Part of SPLASH 2016, Ams- 
terdam, The Netherlands, 30 October-4 November 2016, pp. 430-446. ACM (2016). https://doi.org/10.1145/2983990.2984040

42. Shipilev, A.: The java concurrency stress tests (2018). https://wiki.openjdk.java. net/display/CodeTools/jcstress

43. Steenbuck, S., Fraser, G.: Generating unit tests for concurrent classes. In: Sixth IEEE International Conference on Software Testing, Verification and Validation, ICST 2013, Luxembourg, Luxembourg, 18-22 March 2013, pp. 144-153. IEEE Computer Society (2013). https://doi.org/10.1109/ICST.2013.33

44. Terragni, V., Cheung, S.: Coverage-driven test code generation for concurrent classes. In: Dillon, L.K., Visser, W., Williams, L. (eds.) Proceedings of the 38th International Conference on Software Engineering, ICSE 2016, Austin, TX, USA, 14-22 May 2016, pp. 1121-1132. ACM (2016). https://doi.org/10.1145/2884781. 2884876

45. Terragni, V., Pezzè, M.: Effectiveness and challenges in generating concurrent tests for thread-safe classes. In: Huchard, M., Kästner, C., Fraser, G. (eds.) Proceedings of the 33rd ACM/IEEE International Conference on Automated Software Engineering, ASE 2018, Montpellier, France, 3-7 September 2018, pp. 64-75. ACM (2018). https://doi.org/10.1145/3238147.3238224

46. Vafeiadis, V.: Automatically proving linearizability. In: Touili, T., Cook, B., Jackson, P. (eds.) CAV 2010. LNCS, vol. 6174, pp. 450-464. Springer, Heidelberg (2010). https://doi.org/10.1007/978-3-642-14295-6_40

47. Visser, W., Pasareanu, C.S., Khurshid, S.: Test input generation with java pathfinder. In: Avrunin, G.S., Rothermel, G. (eds.) Proceedings of the ACM/SIGSOFT International Symposium on Software Testing and Analysis, ISSTA 2004, Boston, Massachusetts, USA, 11-14 July 2004, pp. 97-107. ACM (2004). https:// doi.org/10.1145/1007512.1007526

48. Wickerson, J., Batty, M., Sorensen, T., Constantinides, G.A.: Automatically comparing memory consistency models. In: Castagna, G., Gordon, A.D. (eds.) Proceedings of the 44th ACM SIGPLAN Symposium on Principles of Programming Languages, POPL 2017, Paris, France, 18-20 January 2017, pp. 190-204. ACM (2017). http://dl.acm.org/citation.cfm?id=3009838

49. Wing, J.M., Gong, C.: Testing and verifying concurrent objects. J. Parallel Distrib. Comput. 17(1-2), 164-182 (1993). https://doi.org/10.1006/jpdc.1993.1015

Open Access This chapter is licensed under the terms of the Creative Commons Attribution 4.0 International License (http://creativecommons.org/licenses/by/4.0/), which permits use, sharing, adaptation, distribution and reproduction in any medium or format, as long as you give appropriate credit to the original author(s) and the source, provide a link to the Creative Commons license and indicate if changes were made.

The images or other third party material in this chapter are included in the chapter's Creative Commons license, unless indicated otherwise in a credit line to the material. If material is not included in the chapter's Creative Commons license and your intended use is not permitted by statutory regulation or exceeds the permitted use, you will need to obtain permission directly from the copyright holder. 